\title{
Recurrent fractal neural networks: a strategy for the exchange of local and global information processing in the brain
}

\author{
Erhard Bieberich* \\ Institute of Molecular Medicine and Genetics, Medical College of Georgia, 1120 15th Street Room CB-2803, Augusta, GA 30912, USA
}

Received 2 May 2001; received in revised form 11 January 2002; accepted 20 June 2002

\begin{abstract}
The regulation of biological networks relies significantly on convergent feedback signaling loops that render a global output locally accessible. Ideally, the recurrent connectivity within these systems is self-organized by a time-dependent phase-locking mechanism. This study analyzes recurrent fractal neural networks (RFNNs), which utilize a self-similar or fractal branching structure of dendrites and downstream networks for phase-locking of reciprocal feedback loops: output from outer branch nodes of the network tree enters inner branch nodes of the dendritic tree in single neurons. This structural organization enables RFNNs to amplify re-entrant input by over-the-threshold signal summation from feedback loops with equivalent signal traveling times. The columnar organization of pyramidal neurons in the neocortical layers V and III is discussed as the structural substrate for this network architecture. RFNNs self-organize spike trains and render the entire neural network output accessible to the dendritic tree of each neuron within this network. As the result of a contraction mapping operation, the local dendritic input pattern contains a downscaled version of the network output coding structure. RFNNs perform robust, fractal data compression, thus coping with a limited number of feedback loops for signal transport in convergent neural networks. This property is discussed as a significant step toward the solution of a fundamental problem in neuroscience: how is neuronal computation in separate neurons and remote brain areas unified as an instance of experience in consciousness? RFNNs are promising candidates for engaging neural networks into a coherent activity and provide a strategy for the exchange of global and local information processing in the human brain, thereby ensuring the completeness of a transformation from neuronal computation into conscious experience.
\end{abstract}

(C) 2002 Elsevier Science Ireland Ltd. All rights reserved.

Keywords: Networks; Fractal; Brain; Mind; Consciousness; Neuron

\section{Introduction}

In classical neural networks, logical AND operations follow the MacCulloch-Pitts rule: a

* Corresponding author. Tel.: +1-706-721-9113; fax: +1706-721-8765

E-mail address: ebieberich@mail.mcg.edu (E. Bieberich). dendritic input that provokes a particular threshold voltage in or close to the somatic region of a neuron results in the generation of an action 
potential, and thus the transduction of an output signal to the downstream network (Haykin, 1994 Koch, 1999). Most recently, it has been found that dendritic trees are endowed with efficient mechanisms to counteract the attenuation of input signals traveling down to the somatic region (Magee and Cook, 2000; Spruston, 2000). Hence, the strength of a dendritic input at the somatic region is independent of the site of its injection within the dendritic tree. This principle allows for the phasedependent amplification of input signals: they can only contribute to an over-the-threshold summation if they arrive in the somatic region at the same time. Accordingly, their time-of-arrival in the dendritic tree is reciprocally correlated to the distance of their injection site from the somatic region. Distal signals that arrive earlier are integrated with proximal signals that arrive later.

Although this mechanism efficiently amplifies signals that are in phase, it inevitably destroys information that may have been encoded in their temporal and spatial distribution within the dendritic tree: spike trains arriving at different synaptic sites are reduced to a single action potential that departs from the neuron. In a convergent network, this will ultimately result in a tremendous loss of information. This loss can be avoided if the output from a single neuron is fanned out into a divergent downstream network, the signal distribution of which 'reconstructs' the information that has been encoded in the original dendritic input. A reconstruction of information can be achieved if the spatio-temporal distribution of action potentials within a network (neural network activity) is correlated with that of dendritic input by a particular connectivity between network and dendritic tree. Ideally, this connectivity should perform data compression for sparse encoding with minimal loss of information. Data compression compensates for a reduced number of transduction channels in convergent networks and allows for reconstruction of the original data set.

Functional MRI and EEG have shown that cognitive processes are organized in specialized areas or modules that are distributed throughout the brain (Adcock et al., 2000; Greene et al., 2001; Zeki, 2001). The neuronal computation of cognitive processes in remote brain areas, however, raises the question of how the results of these computations are integrated as an instance of experience in human consciousness. The solution of this dilemma demands for a mechanism that fans out a local input into a global neuronal network and, on the other hand, makes a global network output accessible as local input. A potential solution can be found in a neural network with alternating convergent and divergent activity. This concept underlies recurrent neural networks that have been intensively studied by Hopfield, Tononi, and Edelman (Hopfield, 1982; Sporns et al., 1991). In particular, thalamo-cortical loops have been discussed as 'global workspace', in which consciousness arises (Baars, 1997). The output of the global network activity is backlooped onto single neurons within a network, and thus locally accessible. Recurrent connectionist networks, however, leave important questions open: How is a recurrent network correlated with the natural architecture of the brain? How is the recurrent dendritic input phase-locked with the global output? How is the information that is encoded in this output compressed into a limited number of feedback channels? How is the compressed information converted into a complete data set for conscious experience? And as a final challenge posed by these network models: do we need a single master neuron that receives the complete network output in order to generate consciousness?

These are the questions we will approach in the following discussion. A coding pattern will be described as spatio-temporal distribution of address registers with a particular topology. These address registers are correlated with the location of excited synapses (active dendritic spines) or firing neurons (active neurons) within a network. Our working hypothesis arises from the assumption that there is a topological operation that iteratively maps the downscaled version of a global coding structure onto each part of a neuronal network. The topology generated by a contraction mapping operation is termed self-similar or fractal (Barnsely, 1993; Mandelbrot, 1983; Peitgen et al., 1992). In a self-similar structure, there is a particular relationship between the number of sub-structures (or pieces) $m$ and their size reduction $r$ : 
$D=\log (m) / \log (1 / r)$,

$D=$ Hausdorff dimension

A self-similar structure is called fractal if $D$ exceeds the topological dimension. For example, a simple two-dimensional square is self-similar because a partitioning into four $(=m)$ equal pieces always reduces the side length of one piece by $r=$ $1 / 2$. A square, however, is not fractal because $D=$ $2(=\log (4) / \log (2))$, and thus identical to the topological dimension. A seacoast, on the other hand, is self-similar and fractal because $D$ of a one-dimensional coastline is usually equal to 1.2 (Mandelbrot, 1983). The topological advantage of fractals emerges from the optimized covering of a higher-dimensional space with a lower-dimensional structure, e.g. filling of a tissue (two- or three-dimensional) with branched, one-dimensional cellular extensions (e.g. dendrites).

You may zoom into a fractal and you will find the same structure at all scales. This idealized topological operation will be applied to two scaling levels within a neural network. The spatial and temporal distribution of action potentials as neuronal network output will be mapped as recurrent signaling input onto the synaptic sites within the dendritic tree of each neuron. This connectivity allows for self-similar coding: the activation pattern within the dendritic tree is a downscaled version of the global network output. We hypothesize that this network model, which will be termed 'recurrent fractal neural network (RFNN)', arises naturally from the architecture of the brain, self-organizes phase-locking of global output with recurrent local input, efficiently compresses information by encoding of intrinsic selfsimilarity, and engages remote brain areas into a coherent activity without convergence onto a single center of consciousness. We will see that RFNNs efficiently combine recurrent connectionist networks with temporal and dendritic encoding. This type of encoding has been suggested to underlie conscious experience (Orpwood, 1994; Woolf, 1999). We will also apply principles of fractal organization by RFNNs to sub-neuronal structures. After all, consciousness will require a molecular substrate. In Section 3, we will discuss that the topological concept that underlies the generation of fractals reconciles a first-person perspective on the unity of mind with a reductionist approach that dissects the brain into single computational units.

\section{Discussion}

\subsection{RFNNs emerge naturally from the architecture of the brain}

The neuronal architecture, in particular dendritic and axonal branching, has often been described as self-similar or fractal (Caserta et al., 1995; Cannon et al., 1999; Porter et al., 1991). Although these structures are not ideal fractals in a strict topological sense (their fractal dimension may vary within a single neuron), we will discuss that their intrinsic structural organization shows characteristics of fractals that will naturally sustain an RFNN. Fig. 1A shows the columnar organization of the mammalian neocortex. The dendritic and axonal architecture of pyramidal neurons within the layers III and V can be described as tree-like structures. Fig. 1B shows that these neuronal trees may have direct or inverse polarity as determined by the branching progression: dendritic branches and axonal (network) branches become successively shorter or longer (direct polarity), or one type of branches becomes shorter whereas the other one becomes longer (inverse polarity). The task of neuronal trees with direct or inverse polarity is to collect (dendrites) and to distribute (axons) signals while economically covering a maximum of space with a minimum of overlapping. Hence, optimized branching follows the principle of fractality (Mandelbrot, 1983; Turcotte et al., 1998): the number $m$ of dendritic nodes $\mathrm{n}_{i}$ is inversely correlated to the reduction $r$ of branch length $l_{i}$, which can be described by Eq. (1). The self-similarity or Hausdorff dimension $D$ has been found to range from 1.5 to 1.9 for the projections of natural neurons, indicating that neuronal trees with a topological dimension of one (branching) are indeed fractals (Caserta et al., 1995; Cannon et al., 1999).

Fig. 1C shows a model neuron with $D=1.73$ that has been generated by an iterated linear affine 

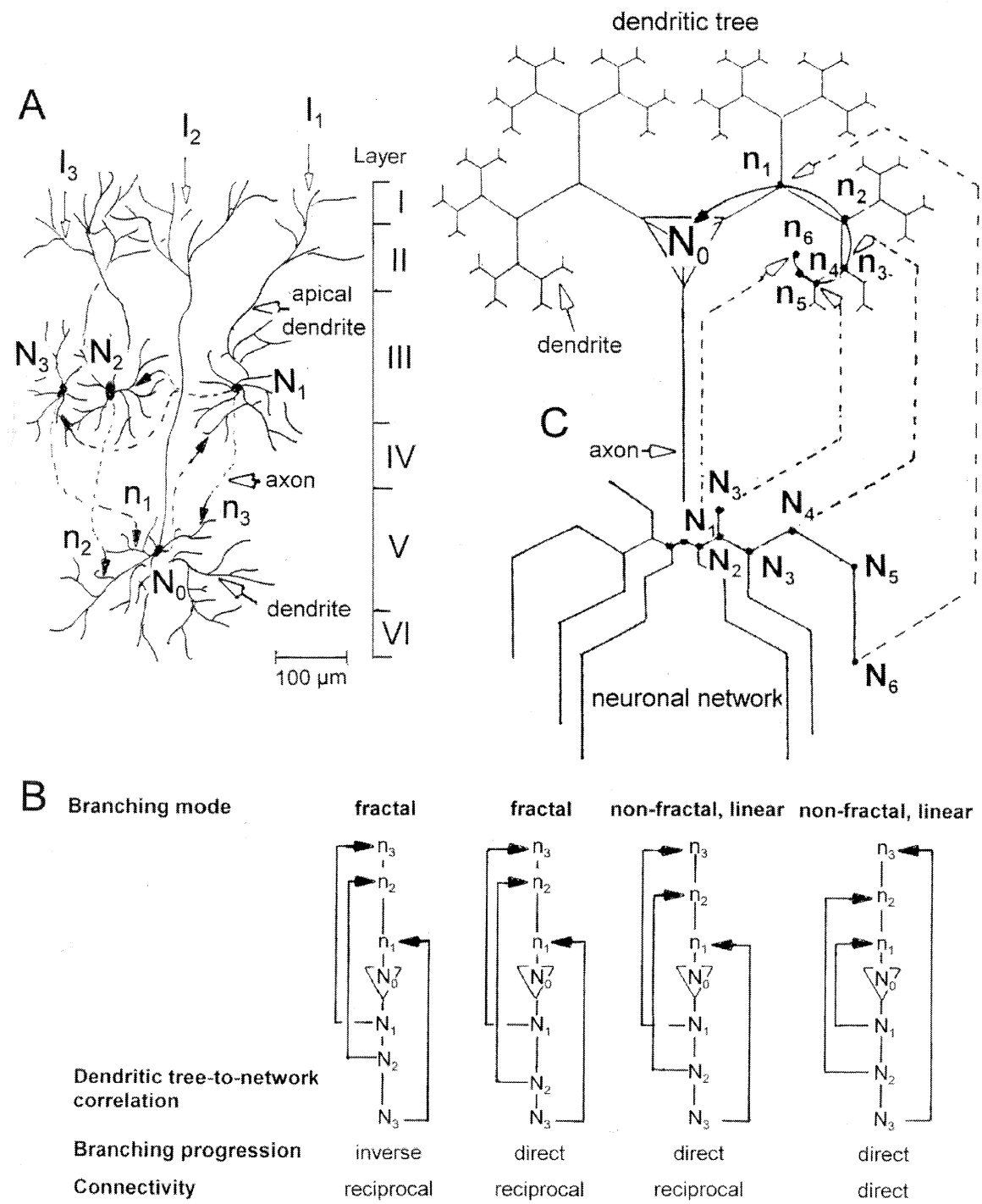

Fig. 1. Morphology and connectivity of RFNNs. (A) Principle of columnar organization of pyramidal neurons in neocortical layers I (cortex surface) to VI (cortex inside). Input $\mathrm{I}_{i}$, from layers I or II is collected by apical dendrites of neurons $\mathrm{N}_{i}$, in layer III, which process and distribute this input to neuron $\mathrm{N}_{0}$ in layer $\mathrm{V}$. Reciprocal feedback loops from $\mathrm{N}_{0}$ in layer $\mathrm{V}$ to $\mathrm{N}_{i}$ in layer III are hypothetical. (B) Different connectivities within RFNNs. (C) Model neuron $\mathrm{N}_{0}$ that receives recurrent input from $\mathrm{N}_{i}$. The branching mode and progression is fractal with inverse polarity (dendritic branches become successively shorter, whereas network branches become longer), the feed back connections are reciprocal (outer branches of the dendritic tree receive recurrent input from inner branches of the network tree). Note, that the dendritic tree in $\mathrm{C}$ is equivalent to that in layer $\mathrm{V}$ of $\mathrm{B}$.

transformation: (i) a linear translation of each new branching point by the rectangular coordinates $x_{n}$, $y_{n}$ of the preceding branch (with its branching point as origin of the coordinate system); (ii) rotation of each new branch by multiples $(k=1$, 2) of $120^{\circ}(=a)$; and (iii) downscaling of the branching length by a reduction factor of $r=0.67$. It should be noted that this type of transformation operation arises naturally from biological growth processes with branching that follow a scaling law. These branching processes have been described as L (Lindenmeyer) or cellular automata systems. 
They yield equivalent fractal structures that are derived from the following operation for iterated linear affine transformation (Mandelbrot, 1983; Peitgen et al., 1992):

$\left[\begin{array}{l}x_{n+1} \\ y_{n+1}\end{array}\right]=r \cdot\left[\begin{array}{cc}\cos k \alpha & -\sin k \alpha \\ \sin k \alpha & \cos k \alpha\end{array}\right] \cdot\left[\begin{array}{l}x_{n} \\ y_{n}\end{array}\right]+\left[\begin{array}{l}x_{n} \\ y_{n}\end{array}\right]$

In fractal trees, Eq. (1) and Eq. (2) can be combined by $D$ according to:

$D=\log (a) / \log (1 / r)$

with $a$, number of $k$ or number of branches/node, in our model $a=2$ (Fig. 1C).

Fig. $1 \mathrm{C}$ shows that this fractal tree-like structure will satisfy the condition of reciprocal signal reentry. A distal axonal node $\mathrm{N}_{j}$ is back-looped to a proximal dendritic node $\mathrm{n}_{i}$ and vice versa. For example, $\mathrm{N}_{6}$ is back-looped to $\mathrm{n}_{1}$, whereas $\mathrm{N}_{1}$ reenters the dendritic tree at $\mathrm{n}_{6}$. Note, that the term 'node' is meant in a broader sense than just the branching point: $\mathrm{n}_{i}$ or $\mathrm{N}_{j}$ are individual addresses within a tree. This may be a dendritic spine that is located within a particular branch, or a neuron along one branch of a neural network. Within a dendritic tree that shows successively shorter branches, the number of spines per branch is inversely correlated to the distance from the somatic region and allows for the recruitment of more synaptic input per branch from a more proximal location. However, we will simplify this model by assuming that the initial, perisomatic branches are not included because of their sparse input, which has often been found to be inhibitory (Megias et al., 2001). Now suppose, that with fast axonal $(\sim 100 \mathrm{~m} / \mathrm{s})$ and relatively slow dendritic $(\sim 1 \mathrm{~m} / \mathrm{s})$ signal transport, the total traveling time for reciprocally connected feedback loops is the same (Agmon-Snir and Segev, 1993; Koch, 1999; Stuart et al., 1997). A distal input arrives earlier, but takes more time to travel down to the somatic region, whereas a proximal input arrives later, but has a shorter traveling time within in the dendritic tree. In RFNNs, proximal and distal input arrives simultaneously at the somatic region and can be integrated for action potential generation.

Fig. $1 \mathrm{~B}$ and $\mathrm{C}$ show the key feature of RFNNs: an address register of $\mathrm{N}_{j}$ neurons within a network is mapped reciprocally onto a downscaled register of $\mathrm{n}_{i}$ dendritic nodes. Thus, the fractal architecture of an RFNN naturally sustains a scale-invariant coding structure: the global coding output at a larger scale (network) is mapped onto a local access site at a smaller scale (dendritic tree). Fig. 1B also shows alternative models that fulfill the reciprocity and downscaling criterion, and hence would sustain a scale-invariant coding structure. The non-fractal (and not self-similar) tree with equal branch lengths, however, will not cope with optimized space filling due to overlapping branching. A special case is the self-similar but nonfractal tree, the branching of which proceeds with a reduction factor that is the exact inverse of the branching number, e.g. $r=0.5$ and $m=2(D=$ 1.0). This tree structure compensates for less optimal space filling by establishing full reciprocity: the number of output loops per network branch will not exceed the number of input loops per dendritic branch. A fractal tree does not show full reciprocity because the sum of the lengths of all branches at any particular step up or down the tree will be different from the sum of other steps. It is quite possible that nature has found a compromise between optimal space filling and reciprocity in RFNNs.

Which architecture of the brain shows characteristics of fractal trees and may be a candidate substrate for RFNNs? Most recently, the evaluation of connectivity in cortical columns of pyramidal neurons in the neocortical layers III and V has shown that layer $\mathrm{V}$ projects a limited number of axons to layer III, but receives a 10 -fold larger number of back-projections from this layer (Thomson and Bannister, 1998). Although there is no direct proof that these projections are connected as feedback loops, they fulfill the structural characteristics to sustain an RFNN. As shown in Fig. 1A, the neurons of layer $\mathrm{V}$ may fan out their action potentials to layer III (divergent phase) and collect the neuronal output from this layer as recurrent input in their dendritic trees (convergent phase). A signal from layer $\mathrm{V}$ could be transduced via fast axonal transport to layer III, back-looped, and integrated with other recurrent input in the dendritic trees of layer $\mathrm{V}$. Fig. 1A shows that this network organization is consistent with the tree structure of pyramidal 

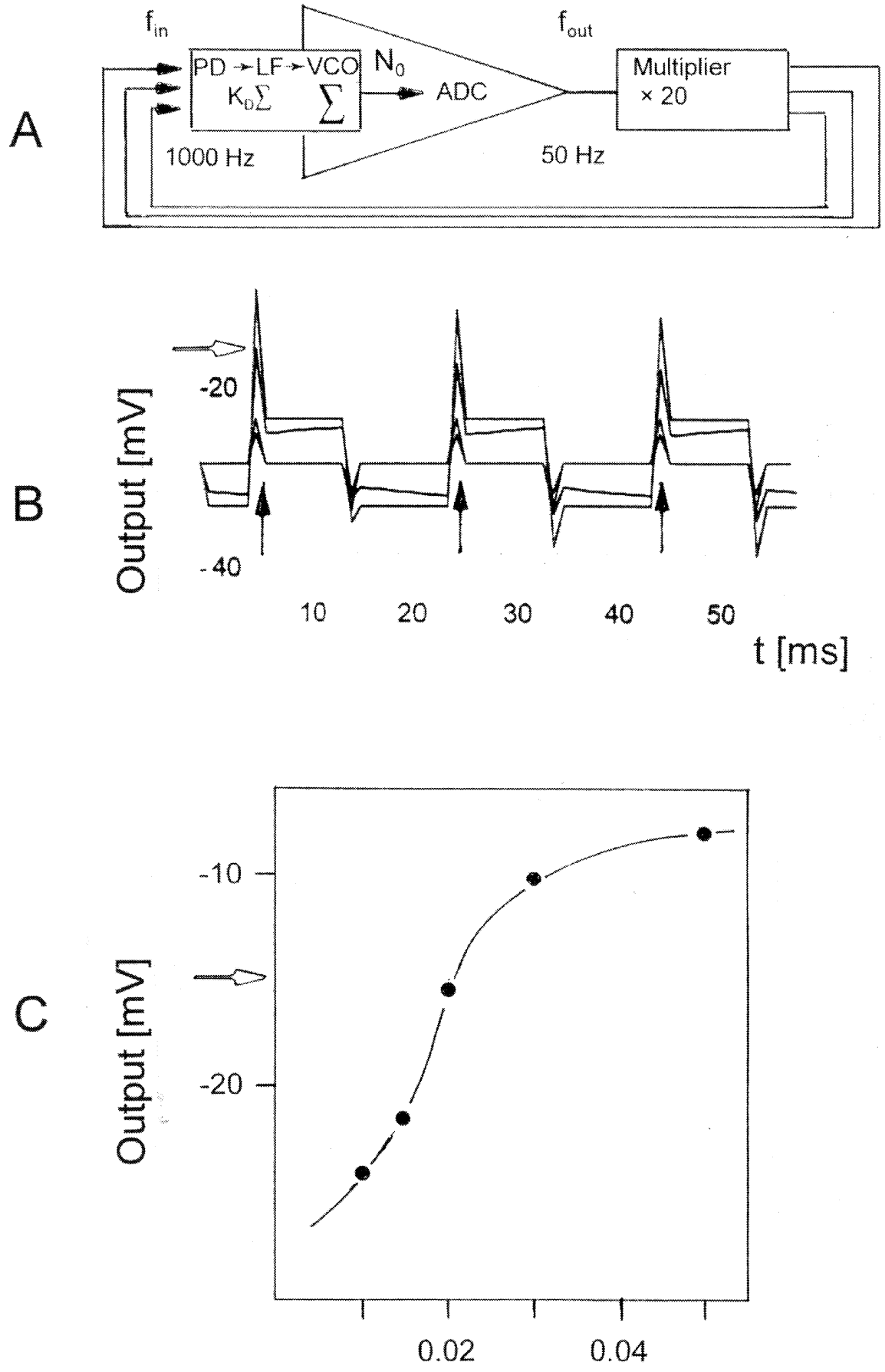

Phase Detector gain [V/rad]

Fig. 2 
neurons and the columnar dimensions within the mammalian neocortex. It also indicates that layer III neurons are equivalent to the downstream network of an RFNN, which is consistent with the experimental observation that they have a higher probability to be connected to neighboring neurons than those in layer $\mathrm{V}$ (Thomson and Bannister, 1998). Layer V neurons appear not to receive direct input from superficial layers but are excited by layer III neurons that may receive input via their apical dendrites in layers I or II.

\subsection{RFNNs self-organize phase-locking of feedback loops}

Neuronal networks are known to spontaneously initiate oscillations at frequencies between 10 and $100 \mathrm{~Hz}$ (Jefferys et al., 1996; Menendez de la Prida and Sanchez-Andres, 2000). This network activity has been ascribed to coupling of neurons by phaselocked loops (PLLs) with each neuron acting as voltage controlled oscillator (VCO, Hoppensteadt, 1989). Within a certain frequency range, VCOs with feedback loops can compensate for a phase shift and pull the system back to its center frequency (Best, 1999). Fig. 2A shows that recurrent neuronal networks fulfill most criteria of PLLs with phase (or coincidence) detector (PD), loop filter (LF), and VCO (Borisyuk and Hoppensteadt, 1998). The exact location of these elements within a neuron is a matter of further investigation. Distal dendrites, however, will certainly act as PDs, whereas the functional equivalent of LFs and VCOs may be located at a site that is more proximal to the somatic region of the neuron. In principle, a PLL works well within the range of $\mathrm{d} \omega / \mathrm{d} t<\omega^{2}$ for a frequency offset $\mathrm{d} \omega$ from the center frequency $\omega$ (Best, 1999). The caveat of a neuronal PLL, however, is its dependence on the broad frequency band of recurrent signals picked up by dendrites. A computer simulation with $\omega$ set to $50 \mathrm{~Hz}$ and spike bursts of $\mathrm{d} \omega=1000 \mathrm{~Hz}$ for dendritic input results in a periodic fluctuation of the PD-output around $\omega$ (Fig. 2B). A neuron could not phase-lock a signal if it works like a completely analog PLL. However, over-thethreshold summation of incoming post-synaptic potentials and threshold-controlled action potential generation endows the neuron with an analog digital converter (ADC) that chops the PLL fluctuation into discrete spikes (Fig. 2B). Vice versa, broadening of a distinct synaptic input while traveling down the dendritic tree converts a digital into an analog signal. As shown in Fig. 2B and C, the output voltage for over-the-threshold summation relies critically on the detector gain $K_{\mathrm{D}}$ (voltage increase/phase) in the dendritic tree. In a neuronal PLL, an action potential is generated if a minimum number of dendritic spikes are in phase, resulting in rapid voltage increase to the depolarization threshold. It has been found that a Hodgkin-Huxley neuron that operates as integrate-and-fire' unit indeed responds with a rapid voltage gain to incoming dendritic spikes at high frequency (Koch, 1999; Koch and Segev, 2000). This observation is consistent with the computer simulation of PLLs as in Fig. 2, and has led to the idea that neuronal networks may function like frequency-modulated VCOs in FM-radios (Hoppensteadt and Izhikevich, 1998).

Can a neuronal PLL drive an RFNN by the recruitment of a particular network, the neurons of which feed the PLL with dendritic input spikes that are in phase? The following analysis will show that a recurrent network with multiple feedback loops will phase-lock a particular RFNN by the recruitment of loops with reciprocal connectivity. Fig. 3 shows a connectivity matrix for RFNNs with dendritic input $\left(\mathrm{n}_{i}\right)$ and neuronal output $\left(\mathrm{N}_{j}\right)$ addresses represented as rows or columns, respectively. The matrix values are the signal-traveling times between $\mathrm{N}_{j}$ and any $\mathrm{N}_{0}$ or vice versa, which

Fig. 2. Simulation of a neuronal PLL. (A) PLL circuit model for neuron $\mathrm{N}_{0}$ in an RFNN; PD, phase detector; LF, loop filter; VCO, voltage controlled oscillator; $K_{\mathrm{D}}$, phase detector gain; ADC, analog digital converter. (B) Linear PLL computer simulation in passive lag mode (program PLL simulation V1.1, Best Engineering, is commercially available through Best, 1999); PLL supply voltage -0.06 $\mathrm{V},+0 \mathrm{~V} ; K_{\mathrm{D}}$, variable, $0.01-0.10 \mathrm{~V} / \mathrm{rad}$; PD saturation voltage, $-0.06 \mathrm{~V},-0.01 \mathrm{~V}$; LF time constants $t_{1}, 0.005 \mathrm{~s}, t_{2}, 0.010 \mathrm{~s}$; VCO gain $K_{0}, 13001 / \mathrm{s} \mathrm{V}$; VCO saturation voltage $-0.06 \mathrm{~V},-0.01 \mathrm{~V}$. (C) Dependence of PD output from $K_{\mathrm{D}}$. An action potential is generated if a certain threshold voltage (arrow in Fig. 2) is reached (not simulated). 
will rely on the particular dendritic node or spine $\mathrm{n}_{i}$ that has been chosen for signal re-entry at $\mathrm{N}_{0}$. For example, the neuron $\mathrm{N}_{1}$ may be connected to $\mathrm{N}_{0}$ via $\mathrm{n}_{1}$ or $\mathrm{n}_{3}$. An action potential generated at $\mathrm{N}_{1}$ will thus take $1 \mathrm{~ms}\left(\right.$ via $\left.\mathrm{n}_{1}\right)$ or $4 \mathrm{~ms}\left(\right.$ via $\left._{3}\right)$ for traveling to the somatic region of $\mathrm{N}_{0}$. If in phase with other signals it will contribute to the generation of an action potential at $\mathrm{N}_{0}$, which is distributed to the downstream network and arrives at $\mathrm{N}_{1}$ to $\mathrm{N}_{4}$ at times indicated in the second matrix. At this point, we do not make a difference between traveling through network interneurons or direct connection of $\mathrm{N}_{0}$ to $\mathrm{N}_{j}$ via axonal branching of $\mathrm{N}_{0}$ (Fig. 3B). The summation of traveling times for signal transport from $\mathrm{N}_{j}$ to $\mathrm{N}_{0}$ and $\mathrm{N}_{0}$ to $\mathrm{N}_{j}$ gives the total traveling times for all possible feedback loops (resultant connectivity matrix). Signals are in phase if the total traveling times for looping are identical. The stable connections are the ones that allow for over-the-threshold summation at $\mathrm{N}_{0}$. In our example, we have set the number of dendritic input signals to four in order to reach a minimum voltage gain for action potential generation. Hence, four feedback connections will be selected at a looping time of $10 \mathrm{~ms}$, which results in a coherent network oscillation at $100 \mathrm{~Hz}$ (solid lines in Fig. 3B). The other connections will not be amplified and just continue to fluctuate randomly or fade out (dashed lines in Fig. 3B). As shown in Fig. 3A, the final connectivity matrix for stable feedback loops can be translated into binary $x / y$-distribution matrices that denote the addresses of active neurons in the network and those of active spines in the dendritic tree (Fig. 3B). Once the network shows coherent oscillation, the frequency offsets are small and the PLL can pull the system back to its center frequency. From this simple example, it can be seen that RFNNs can stabilize neuronal network oscillations by self-organized recruitment of reciprocal PLLs.

\subsection{RFNNs compress information by robust encoding of self-similarity}

It has been shown that individual neurons in the temporal cortex of macaque monkeys respond to different images with intrinsic self-similarity and identical complexity, irrespective of their shape, color, or size (Miyashita et al., 1994). This observation indicates that neuronal networks may recognize images by utilizing intrinsic selfsimilarity for sparse encoding of a pixel distribution. Information compression, in particular of images, is certainly required at some point during neuronal computation with convergent signaling pathways. In simple words, a small number of action potentials should encode a large number of pixels. This demands for an appropriate transformation function for sparse encoding of a spatial pixel distribution. Fractal image compression has been used successfully in art and information technology (Barnsely, 1993; Peitgen et al., 1992). It is based on encoding of shape and contrast by a linear affine transformation that correlates the self-similar parts of a picture with each other. Ideally, this transformation is part of an iterative functional system that encodes six numerical parameters for shapes in the $x / y$-plane (two for translation, rotation, and size reduction; Peitgen et al., 1992). Fig. 4A shows how a fractal pixel distribution can be used for scale-invariant encoding of signal patterns. The matrices show pixel distributions for $\mathrm{Y}$ or triangle-shaped contours that repeat themselves on two different scaling levels termed 'global' and 'local'. The transformation function can be obtained in two ways that are interconvertible. In the first method, usually applied for fractal image compression by encoding of global-to-local self-similarities, a coarse overall structure is downscaled (in our example 9:1) and shifted over the image by successive translation and rotation. A match yields the parameters for a linear affine transformation that is similar to the one already used for the generation of fractal tree structures (Eq. (2)).

The second method is based on the globalization of a local signal distribution resulting in an enlarged image with intrinsic self-similarity. The numerical solution for this globalization can be achieved by calculation of the Kronecker square matrix product $\left(M^{2}\right)$ that has been derived from the local signal distribution as shown in Fig. 4A (Gazale, 1999). In our example, the coarse distribution of eyes, nose, and mouth constitutes four input signals that are fed into nine cortical 


$$
\begin{array}{ccc}
t\left(N_{j} \text { to } N_{0}\right) & t\left(N_{0} \text { to } N_{j}\right) & \text { t looping } \\
{[\text { msec] }} & {[\mathrm{msec}]} & {[\mathrm{msec}]}
\end{array}
$$

$\begin{aligned} & N_{1} \\ & N_{2} \\ & N_{3}\end{aligned}\left[\begin{array}{cccc}2 & 0 & 8 & 0 \\ 2 & 6 & 12 & 0 \\ 2 & 6 & 12 & 20 \\ 4 & 10 & 16 & 0\end{array}\right]+\left[\begin{array}{llll}2 & 2 & 2 & 2 \\ 4 & 4 & 4 & 4 \\ 4 & 4 & 4 & 4 \\ 6 & 6 & 6 & 6\end{array}\right]=\left[\begin{array}{cccc}4 & 2 & 10 & 2 \\ 6 & 10 & 16 & 4 \\ 6 & 10 & 16 & 24 \\ 10 & 16 & 22 & 6\end{array}\right]$

A
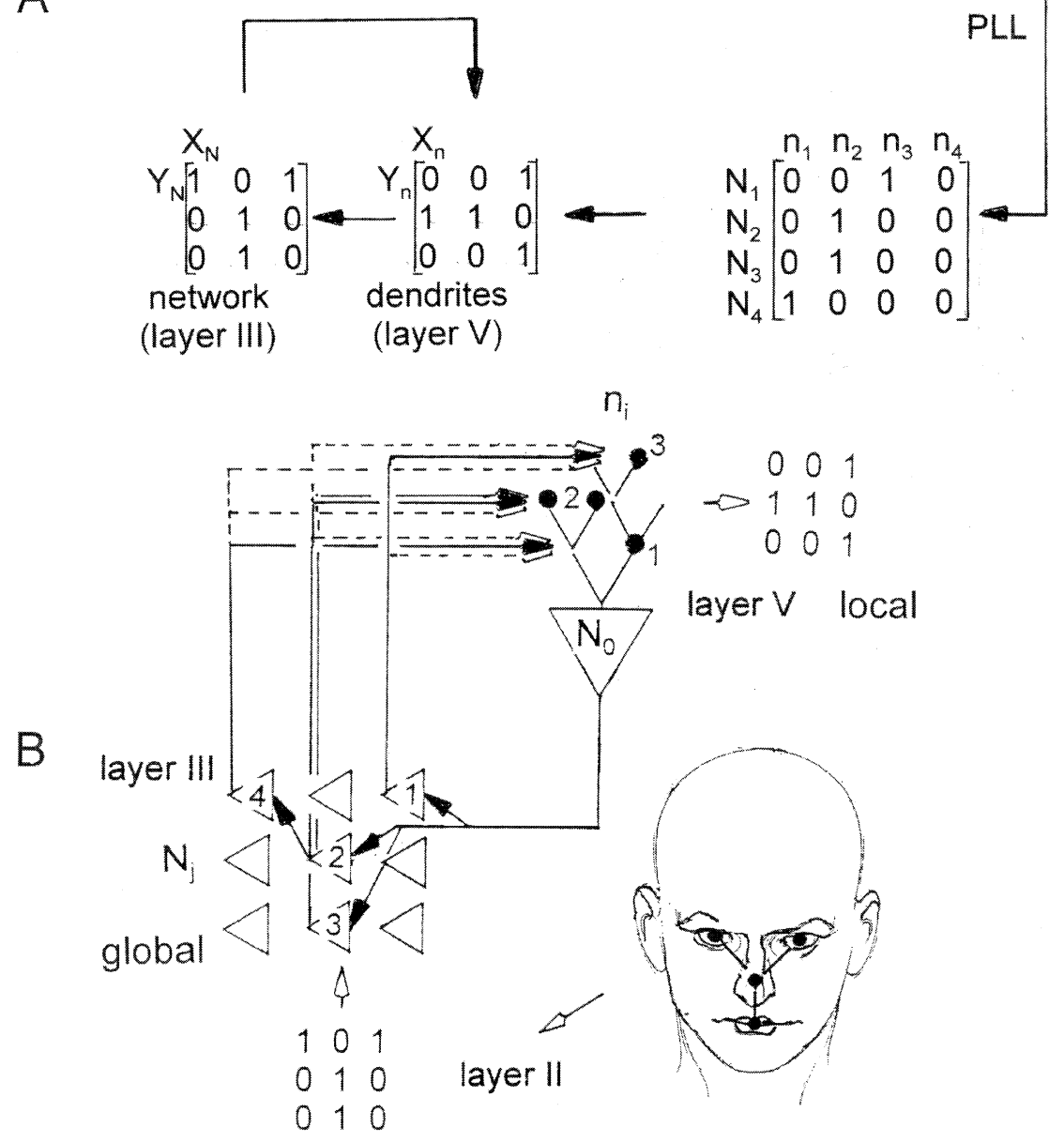

Fig. 3. Input-output analysis for selection of stable signal loops in an RFNN. (A) The left connectivity matrix shows the time that is required to travel from $\mathrm{N}_{j}$ back to the somatic region of $\mathrm{N}_{0}$ via re-entry at the dendritic node (spine) $\mathrm{n}_{i}$, the matrix in the middle shows the time an action potential needs to travel from $\mathrm{N}_{0}$ to $\mathrm{N}_{j}$. The resultant matrix shows the total looping times for any connection between $\mathrm{N}_{0}$ and $\mathrm{N}_{j}$. Loops are amplified (value 1 in bottom connection matrix) if they generate an action potential at $\mathrm{N}_{0}$ whereas the others are attenuated (value 0 ). The connection matrix can then be transformed into $x / y$-distribution matrices showing the spatial coding structure for network (global, layer III) and dendritic (local, layer V) activity. (B) Integration of the matrix model and the neocortical column architecture into vision coding by an RFNN. A Y-manifold detects the coarse features of a human face (eyes, nose, mouth) and translates these into an $x / y$-distribution matrix that activates the network neurons $\mathrm{N}_{j}$ in layer III (global). The global network activity is back-looped onto the dendritic tree of $\mathrm{N}_{0}$ in layer $\mathrm{V}$ (local). The local input is processed by the PLL characteristics of $\mathrm{N}_{0}$ as described in (A), and the computational result fanned out onto the network until stable signal loops (orbits) are selected. 


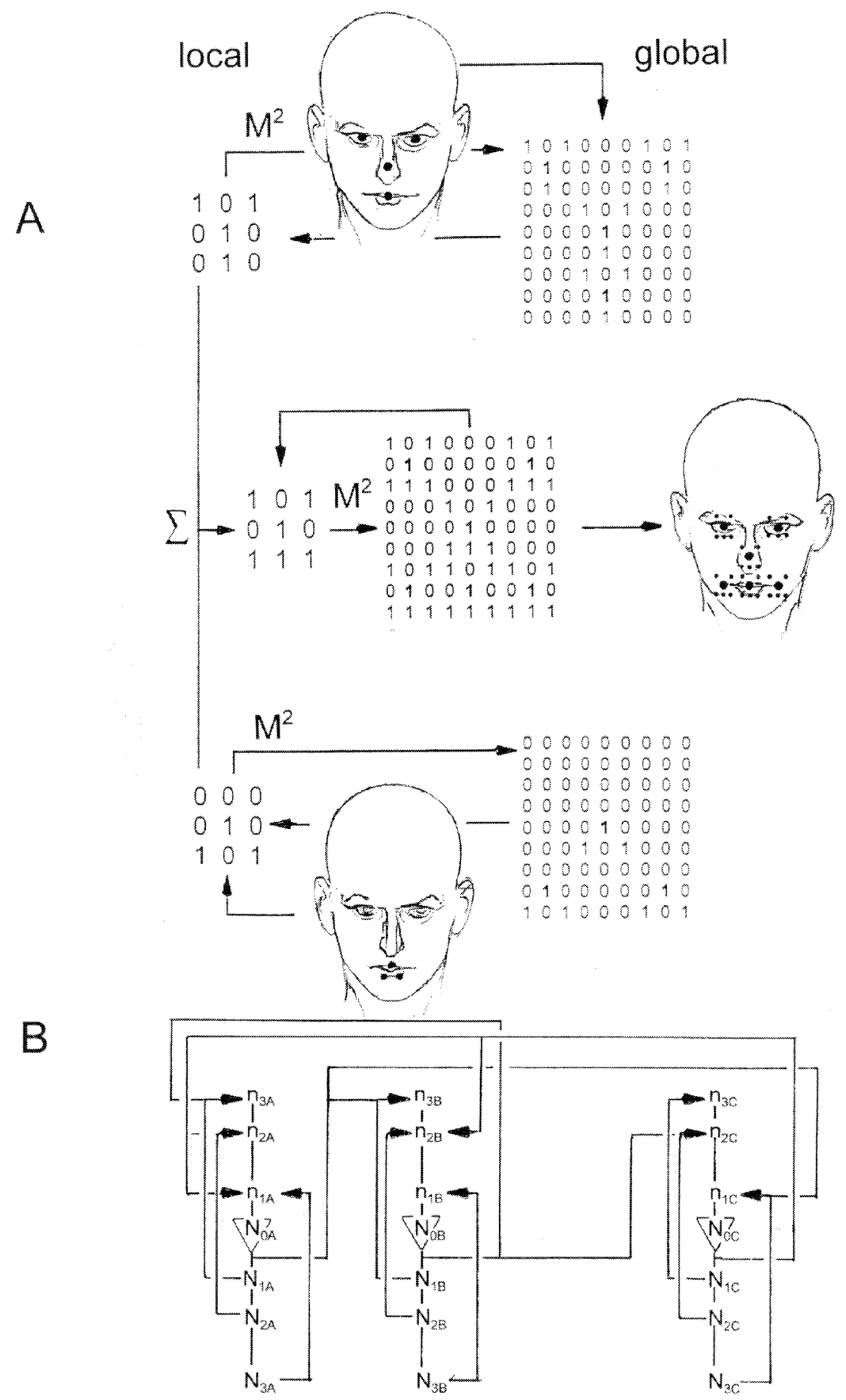

Fig. 4

columns. Fig. 4B shows that the columns form an extended RFNN, which results in fractal mapping of the original input (global 1) onto each column (local 1). Smaller features of the face, e.g. the pixel distribution of the lip contour, are fed into one column (global 2), which gives rise to a down- 
scaled input map within the dendritic tree of each neuron (local 2). This dendritic input is superimposed with that arising from processing of the coarse features. The resulting dendritic input (local 3 ) is now fanned out into the entire array of cortical columns giving rise to a face-like pictorial with intrinsic self-similarity (global 3). Each scaling level of an RFNN (dendritic tree, column, or array of columns) encodes a manifold or transform (e.g. triangle or Y) that can be up- or downscaled and matched with scale-invariant features in the image. This process is equivalent to established methods of image compression using sets of fractal transforms for feature correlation (Peitgen et al., 1992). In accordance with these methods, the compressed set of data (e.g. the triangle or Y-manifold) is not identical to the final image but contains the entire information for its reconstruction. This has already been shown to provide sparse encoding of pictures that are far more complicated and contain more subtle details than those in Fig. 4A. Another important feature of fractal encoding is its robustness and resistance toward local disturbances, e.g. by signal noise, which results from a self-similar coding structure at two scaling levels.

As indicated in Fig. 3B and Fig. 4A, the response of the networked neurons to particular features is rather orientation-sensitive, but invariant to size or spatial distance. Interestingly, these predicted characteristics are similar to those found in studies that recorded the single-cell responses in columns of the visual cortex toward stimuli in their receptive fields (DeAngelis et al., 1999; Dragoi and Sur, 2000). It has also been shown that the activity of cortical columns and the spatial integration of remote areas within the visual field are modulated by the surroundings of the classical, centered receptive field. This observation is consistent with a transformation function that integrates peripheral and central substructures (Castet and Zanker, 1999). The characteristics discussed for the visual cortex have been explained with various neural network models that are not necessarily RFNNs (Dragoi and Sur, 2000; Zeki, 2001). However, RFNNs have additional properties that facilitate the exchange of global and local signal processing, which is not shared by models confined to cortical columns or alternating divergence and convergence within feed-forward networks.

\subsection{RFNNs make a coherent, global network activity locally accessible throughout the brain}

In the preceding section, we have discussed that the integration of self-similar features within an image can be achieved by a meta-coding transformation function that reduces redundancy by compression of self-similarity. The Kronecker square product matrix has been used to generate a fractal pixel or signal distribution from a local input. As shown in Fig. 4B, this product matrix is not just a mathematical curiosity, but represents the addresses of dendritic input nodes and those of the neurons participating in the network within each submatrix. Hence, the signal distribution of the network is an up-scaled version of the dendritic input in each of the networked neurons. The relative positions of the input addresses are identical and match with the overall, reciprocal distribution of the neurons (Fig. 4B). These positions are calculated from a fractal distance function that scales the distances between two addresses by a particular scaling factor, i.e. $r=$ 0.67 (Fig. 1C). Within a single neuron (of neocortical layer $\mathrm{V}$ ), the input addresses are equivalent to the location of dendritic spines that are excited by

Fig. 4. Interchange of global and local information processing for sparse encoding of self-similarity. (A) Coarse features of the human face as shown in Fig. 3B activate a global activity pattern in a column array (bold numbers in matrix). Convergent back-looping onto the dendritic addresses of $\mathrm{N}_{0}$ in each column gives rise to a fractal matrix, that is equivalent to the Kronecker square product $\mathrm{M}^{2}$ of the local input matrix (global 1 to local 1). Individual features, e.g. the lip contour, are first locally encoded and then fanned out into the neuronal network (local 2 to global 2). A summation of local dendritic inputs and the Kronecker square product operation integrate self-similar features of the coarse and the fine structure, giving rise to a self-similar face pictorial (local 3 to global 3). (B) Full connectivity between three neocortical columns, which is suggested as computational substrate for A. The complete operation requires nine columns (not shown). 
incoming action potentials. On a larger scale, they correspond to active neurons (of layer III) within a cortical column, but they may also be distributed over remote areas of the brain.

Further, adjacent or nearby columns may be connected by shared neurons for recurrent input, similar to the example for the integration of the triangle and Y-manifold in Fig. 4A. This would necessarily entail the harmonic coupling of RFNNs with the result of a coherent, electrical network activity. Recently, the recurrent connectivity in rat hippocampal slices has been discussed to mediate a spontaneous, frequency-dependent synchronous network oscillation (Menendez de la Prida and Sanchez-Andres, 2000). The coherent coupling of neuronal activity has been suggested to select the neurons that participate in the generation of consciousness (Singer, 2001). Interestingly, EEGs monitoring the electrical activity of the brain have been found to contain a fractal distribution of spike trains with long-range correlations (Likenkaer-Hansen et al., 2001; Teich et al., 1997). Taken together, these observations indicate that a coherent network behavior in the human brain may arise from fractal coding and activity patterns that are initiated during conscious perception.

Coherence has been explained by other network models without necessarily invoking RFNNs. Most of these models, however, do not specify how the firing in a certain phase will transport information between different parts of neuronal networks, or in other words, make the global output accessible locally. In distributed RFNNs, coherence of networks with shared input neurons indicates that at least some dendritic input in each neuron is identical. Hence, coherence of RFNNs results in the global meta-coding of local information, and the local access to its global processing. This interdependence appears to be a prerequisite for the integration of distributed information into an instance of conscious experience.

\section{Conclusions: RFNNs and consciousness}

RFNNs have been discussed as reciprocal PLLs with inverse output-to-input address registers.
Ideally, the distance function for single addresses is fractal and can be used for sparse encoding of self-similarity, and thus, robust data compression within a convergent signal flow by contraction mapping. These characteristics have been shown to reconcile a network structure with its function, even if the structure is reciprocal and inverse, but not ideally fractal. We have also discussed that a local dendritic input can recruit a downstream network processing and that the global network output is readily accessible to each neuron within this network. In analogy to conventional image compression, the brain may use fractal data compression in RFNNs in order to cope with downscaling of coding patterns in a convergent signal flow. We have seen that some of these characteristics can be explained by alternative network models, but not within the range of completeness that is offered by RFNNs. In the following discussion, we will analyze how RFNNs can reconcile the topology of brain and mind, and how they may compress information into a molecular substrate for the generation of consciousness.

To date, modern neuroscience has failed to explain the emergence of the mind from the activity of the brain (Chalmers, 1996). This is a serious setback since it is not very likely that our understanding of consciousness will be enlightened substantially by the discovery of novel neurotransmitters or receptors. Consciousness is just not countable in molecules or genes. We are not even addressing the problem of qualia or perceptual experience, we are just naively asking: Where am I and the world I see and feel in my brain? This fundamental question has been asked numerous times throughout the history of human mankind. Its currently valid version is still the one posed by the French philosopher Rene Descartes in the 17th century: (i) if the mind is a physical entity, it must have a location; and (ii) if it has a location, the topology of the mind must be embedded into the structure of the human brain. Descartes was quite ahead of his (and our) time by recognizing that: (i) if the mind is a physical event it will arise from local effects in the brain; and (ii) local effects are separated in space and time, and therefore, they cannot account for a simultaneous and holistic 
experience of mental images. It may sound paradoxical, but Descartes should have stated: 'I think thereof I cannot be', at least not if we seek to explain consciousness as physical 'being' on the basis of our present knowledge.

Descartes attempted to resolve this paradox by conceiving the Homunculus concept, a fixed focus point of consciousness, the location of which he assumed to be in the pineal gland. Secondly, he assumed a dualistic view in that the mind as mental substance is different from the physical processes affected by it (Chalmers, 1996). These approaches appear naive from our perspective of modern neuroscience. The underlying concept, however, is still valid: (i) a neural network must be organized in such a way that it renders a divergent, globalized network processing of information locally accessible by a convergent signal transport; or (ii) as alternative, neuronal units must share this information at a distance without exchange by classical signal transport channels.

Current neurophysiological concepts assume that consciousness emerges from a coherent activity of neurons observable as $40 \mathrm{~Hz}$ gamma oscillations in the electrical brain activity (Jefferys et al., 1996; Singer, 2001). This is certainly consistent with the convergence approach, but does not explain the coding and transition into an instance of experience that is unified in space and time. Switching two radios on and off at the same time does not make them share their private information if they are tuned onto different frequency sources. On the other side, popular unifying field theories of consciousness appear to thrive on the approach of sharing information at a distance, in particular by electromagnetic fields (Lindahl and Arhem, 1994; John, 2001; Searle, 2000). Unfortunately, these models widely overlook the fact that extracellular field potentials are physical entities that are as local as neurons (Triffet and Green, 1988). Besides the conceptual intricacies arising from the attempt to reconcile these field models with classical neuronal signal processing, brain-wide field models do not solve the binding problem. Information exchange is faster than with action potential transport, but it still needs a time-delayed signal transport by an electromagnetic wave. Imagine two distant radios, e.g., one located on earth and the other one on the moon. These radios do not simultaneously receive the same information even if they are tuned onto the same frequency source. Escaping this locality by a field model would require a radical reformation of physical concepts comparable to observations in quantum theory, in particular EinsteinPodolsky-Rosen type phenomena (Penrose, 1994). It is just the non-local sharing of information experienced from a first-person perspective, which is irreconcilable with local and private information processing by distant neurons or classical field potentials. However, globally distributed information is locally accessible if the structure of the whole is encoded in each of its parts. It is this concept of fractality that makes RFNNs attractive if we seek to reconcile the structure of the brain with the topology of the mind.

In the following analysis we will discuss that the concept of fractality can be equally useful for a description of neural networks and the topology of the mind. As shown in Fig. 5A, we will claim that single percepts, e.g. the visual objects A and B, are distributed in a single mind space (or endo-space) $\mathrm{S}$ that has to be reconciled with physical brain space. Further, we assume that there is a product space $\mathrm{S} \times(\mathrm{A}+\mathrm{B})$ that cannot be resolved into $\mathrm{S}_{1} \times \mathrm{A}+\mathrm{S}_{2} \times \mathrm{B}$ or else it will be split into subspaces $\left(\mathrm{S}_{1}, \mathrm{~S}_{2}\right)$. Imagine the mind would be water in a jar. If you pour the water into two glasses, the sub-spaces, you will split the mind. If separate subspaces existed, they would be separately perceived, which is not the case. The question is: how can $\mathrm{S} \times$ $(\mathrm{A}+\mathrm{B})$ be processed in separate neurons without being split? We will approach this problem by assuming that there is a function $(f)$ for which the following holds: A does not equal B, but $f\left(\mathbf{S}_{1}\right)$ equals $f\left(\mathrm{~S}_{2}\right)$. This is possible if $\mathrm{S}_{1}$ contains $\mathrm{S}_{2}$ as sub-space and vice versa (Fig. 5A, bottom part). In this case, the distribution of nervous signals onto separate neurons does not split $\mathrm{S} \times(\mathrm{A}+\mathrm{B})$ because the product spaces $f\left(\mathrm{~S}_{1}\right) \times \mathrm{A}$ and $f\left(\mathrm{~S}_{2}\right) \times \mathrm{B}$ are distinct, but contain each other. Is there a function on $\mathrm{S}$ that can do the trick? We have seen in Section 1 that fractals are topological objects that fulfill the criterion of containing the whole in each part. This topology warrants that $A$ (in $S_{1}$ ) 


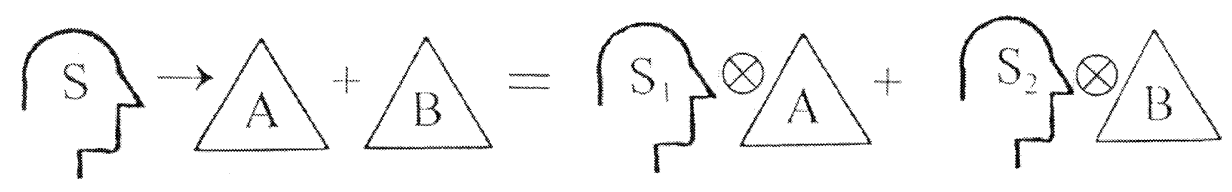

A
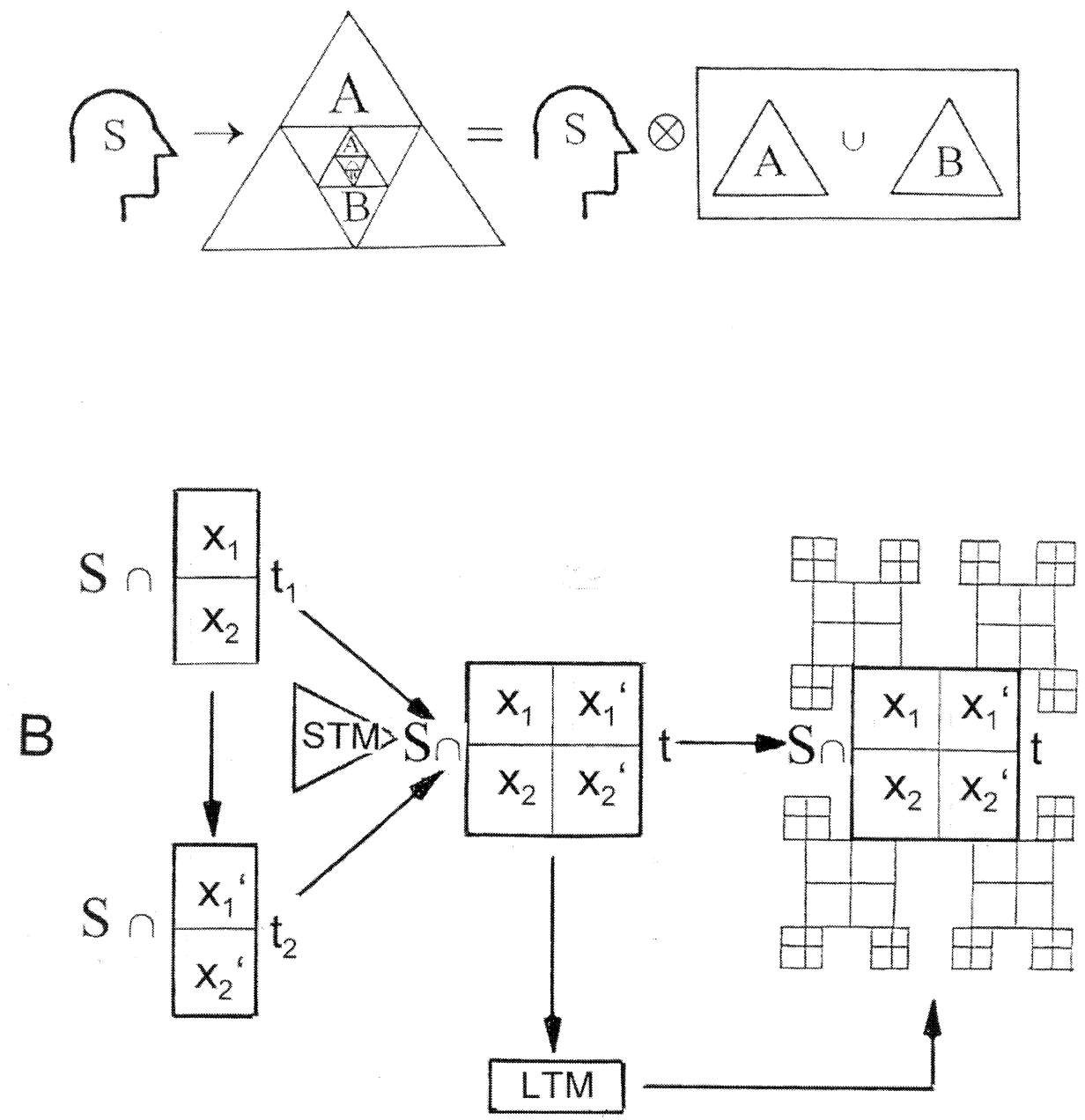

Fig. 5. Fractal topology of the mind. (A) The correlation of an inner space (endo-space) $\mathrm{S}$ with two distinct visual objects A or B splits consciousness into $S_{1} \times A$ and $S_{2} \times B$ if computed with separate neurons and no information exchange. Preservation of an undivided consciousness demands for a complete exchange of information between any two neurons. This is achieved by contraction mapping resulting in fractal embedding of $S_{1} \times A$ and $S_{2} \times B$ into each other's coding structure. (B) Several levels of multi-fractals for embedding of different visual objects at different times $(t)$ into the personal history of the inner observer. This gives the impression of a continuous consciousness with a feeling of identity. STM, short-term memory; LTM, long-term memory.

and $\mathbf{B}$ (in $\mathbf{S}_{2}$ ) are distinct, but $f\left(\mathbf{S}_{1}\right)$ equals $f\left(\mathbf{S}_{2}\right)$ because each sub-space contains the other one as a part. Hence, a fractal product space $\mathrm{S} \times(\mathrm{A}+\mathrm{B})$ could be distributed into separate neurons without 
being split. This may result in a one (observer)-toall (information) correlation as experienced in human consciousness.

Although we do not discuss the physical nature of perceptual experience, sensation, or emotion themselves, we will assume that consciousness is not an independent entity but a quality inevitably arising from an appropriate physical process (Chalmers, 1996). The inner observer is not only aware of outside objects, but does also observe him/herself. The mind within the brain is selfconscious. According to the model of RFNNs, this requires the inclusion of additional information in a multi-fractal coding structure. As shown in Fig. $5 \mathrm{~B}$, the self-awareness of the inner observer as continuous entity emerges from the confrontation of the present with the past in a common fractal space-time. This confrontation may also account for the conscious experience of sensations with duration, e.g. the experience of sounds and music. The integration of one's personal history in a multi-fractal coding structure may be required in order to maintain a feeling of identity: the inner observer must be fed with one's memory in each moment of self-conscious experience. Hence, an RFNN may generate a multi-fractal, the structure of which is modulated by one's personal memory. The generation of fractally structured memory has been suggested to underlie cognitive processes and memory retrieval, and is supported by recent analyses correlating the probability of learning with a fractal time series in the electrical brain activity (Rucker, 1987; West, 2001).

In addition to the previously discussed topbottom line of arguments, we have seen that the feeding of nervous signals into single neurons will require the analysis of molecular mechanisms that underlie the interfacing of physical signaling and mental experience. Although we are certain that the world we experience fits into an organ of the size of a small melon, we feel uneasy about fitting it into a single cell. Size obviously matters when it comes to neuronal signal processing. However, there is no reason to stop at the scaling level of neuronal cells. Eventually, the transition of physical effects into mind experience requires a molecular substrate. In RFNNs, this substrate will gain access to the complete network information only via synaptic input channels of individual neurons. Do these channels have the capacity to transmit the vast amount of information that results in conscious experience?

Assume that the simultaneous arrival of 5000 synaptic input bytes (5000 active spines in the dendritic tree) can be turned over with a frequency of $50 \mathrm{~Hz}$ and encodes information with a compression factor of 20, a factor easily achieved by fractal image compression (Peitgen et al., 1992). A single neuron would receive $5 \mathrm{MB}$ per second, an amount of information that may provide enough high resolution tif-pictures as experienced in human consciousness in this period of time. In other words, a ZIP disk could contain 20 seconds of consciousness. The compression factor is even higher by magnitudes (more than 1000-fold) if a hybrid of fractal and wavelet compression is applied (Davis, 1998). This type of compression would enable the encoding of $5 \mathrm{MB}$ tif-images per second with 100 dendritic spines at a turnover frequency of $50 \mathrm{~Hz}$. In this respect, we should not neglect that during continuous perception, the amount of newly received information per time frame is relatively low and sudden changes in a visual scene are often overlooked (change blindness). With changing distance from a visual object, the self-similarity is already preprogrammed by the temporal integration of consecutive pictures. Hence, it appears not to be impossible to compress the neural network information that underlies the generation of consciousness into single neurons. We may also consider that the efficacy of particular compression methods used for high resolution imaging of brain activity, e.g. fractal analysis of EEGs, indicates that the brain itself may rely on these compression methods for maintaining its activity during conscious perception (LikenkaerHansen et al., 2001; Nan and Jinghua, 1988).

If consciousness may happen in single neurons, why is the brain so big? An answer can only be attempted by assuming that a large amount of the brain is rather busy with pre- or post-conscious processing of information and its preparation for convergent signaling. In other words, the brain is so big because it needs its computational power to compress information into single neurons. The relationship of 10 billion neurons to some hundred 
of single neurons receiving the back-looped information of RFNNs may just be necessary in order to increase the number of potential loops, from which a selection can be chosen to stabilize signal orbits that entertain a continuous consciousness. Fractal attractors are known to stabilize stochastic and chaotic systems (e.g. hurricanes) and have been suggested to select robust signal orbits in the olfactory bulb and the visual system (Freeman and Baird, 1987; Freeman, 1994). Hence, they may also be used to channel a signal flow into single neurons. Each neuron in an RFNN may be the limit point for a convergent sequence of signals with the inner observer located as fixed point where the sequence converges. In a fractal attractor, the location of the inner observer can readily and instantaneously switch between single neurons along with an event stream of experiences in consciousness. This view is in line with the 'global workspace' model, which assumes that consciousness is a receiver of a massively parallel signal sequence with dynamical location (Baars, 1997). It is also consistent with the 'holographic paradigm' in that each part of an RFNN contains the information of the whole structure (Gabor, 1968a,b; Longuet-Higgins, 1968; Pribram, 1982). In fact, RFNNs may revive the discussion on holographic signal processing by using a fractal signal path to generate a holographic imprint or record in each neuron of the network. It has been shown that fractal structures can be transformed into holographic imprints that themselves are fractals (Psaltis et al., 1990; Reiter, 1994). In conclusion, RFNNs are compatible or even equivalent to recent concepts in consciousness research and neural network processing, however, with the advantage of being naturally implemented by the intrinsic architecture of the nervous system.

Once the activity of a neuronal network has been downscaled into the dendritic tree of single neurons, the question arises where the bottom line of data compression is reached. Theoretically, contraction mapping should be an infinitely iterated process until the information is contained within a single limit point. This cannot be achieved readily with RFNNs or any other network theory. After all, we need to get comfortable with a particular state of matter that reconciles an observer singularity with spatially extended neuronal and molecular information processing. On the molecular level, lattice structures have been described to self-organize a spatially extended fractal of orientation or spin states (Mandelbrot, 1983; Schroeder, 1991). It has been found that at exactly body temperature, the neuronal membrane undergoes a sharp transition into an ordered, liquid crystalline lattice. Molecular interactions, e.g. hydrophobic or van der Waals interactions between membrane lipids, may be programmed by incoming synaptic signals that modulate the shape of a spatially extended, fractal lipid lattice or microdomain (Bieberich, 2000a). Recently, it has been found that biological membranes exhibit requisite characteristics of fractal geometry with respect to curvature, shape, and internal molecular organization (Hoop and Peng, 2000; Rabouille et al., 1992; Sugar et al., 2001). Alternatively, microtubules or other filaments of the cytoskeleton may also form a lattice structure that can be shaped into a fractal. This assumption is supported by recent analyses of their fractal architecture (Dufort and Lomsden, 1993). In this respect, it should be noted that neuronal membranes or microtubules have already been suggested as candidate substrates for consciousness (Beck and Eccles, 1992; Bieberich, 2000a; Hameroff 2001; Hameroff et al., 2002; Jibu et al., 1994).

The location of activated dendritic spines may translate the fractal excitation pattern within the dendritic tree into an address register for a fractal membrane or cytoskeleton lattice. In the simplest case, two spike trains meeting at a branching point while traveling down to the somatic region may encode a $x / y$-distribution matrix for programming or modulation of spin or orientation states within a molecular lattice. As discussed in a preceding section, fractal wavelet compression may encode a large amount of information by a limited number of synaptic input, which activates only a small part of the neuron, e.g. dendritic branches or the axonal trigger zone. Interestingly, wavelets are scaled functions that are generated by iterative functional systems similar to those used for the generation of fractals (West, 2001). The combination of wavelets, fractals, and holograms provides 
a whole-in-each-part correlation while avoiding the idea of brain-wide field states controlling individual action potentials. Field states that are deemed important for the generation of consciousness may still exist on a molecular scale, e.g. electrical field potentials on the neuronal membrane surface that guide the orientation and van der Waals interaction of lipids within the membrane bilayer (Radhakrishan and McConnell, 1999). In RFNNs, the shaping of a fractal (membrane, cytoskeleton, or field) lattice within the dendritic tree or close to the somatic region may prevent or facilitate signal transduction via generation of action potentials. Our own, preliminary experimental studies have shown that a molecular template with fractal structure can stabilize intermolecular binding states better than a non-fractal structure (Bieberich, 2000b). The stability of a particular lattice structure, the molecular interactions of which are guided by its fractal geometry, may thus control signal transduction, e.g. by modulation of signal attenuation, slightly shifting the depolarization threshold, or affecting synaptic vesicle formation and transport. The activity of a molecular fractal before generation of an action potential is certainly desired since it may endow us with the opportunity to affect signal transduction in dependence on conscious experience. In conclusion, RFNNs are compatible with the transformation of neuronal into fractal, molecular computation. A detailed analysis, however, will certainly be beyond the scope of the present study.

It is likely that a theory on interfacing of brain and mind in RFNNs will reveal itself as a series of transformations for the contraction mapping of coding structures at different scales: (i) a reciprocal connectivity matrix as shown for the selection of feedback loops (scaling level neuronal network to dendritic tree); (ii) a fractal coding matrix for the spatio-temporal distribution of neuronal and molecular activity (scaling level dendritic tree to molecular lattice), and (iii) a yet unknown matrix operation that creates the consciously perceived world from a molecular activity (interfacing of molecular and mental activity). This activity may rely on the formation of particular fractal field or molecular lattice states in the neuronal membrane or cytoskeleton. Although highly speculative and controversial, the respective final matrix operation may be correlated with the matrix mechanics used to describe superimposed or entangled quantum states (Duggins, 2001; Penrose, 1994). A discussion of superposition states in the human brain is certainly of epistemological value, although their physical existence has been disputed or even excluded due to rapid decoherence in a wet, warm environment of brain tissue (Seife, 2000; Tegmark, 2000). Our own experimental results with fractal and non-fractal model templates have shown that the persistence of a quantum coherent state can be prolonged at ambient temperature if the molecular substrate has fractal geometry (Bieberich, 2000b). However, a potential significance of fractal lattices for the emergence of consciousness from a molecular quantum computation is very hypothetical and deserves an analysis that should be separated from the discussion of RFNNs.

Molecular operations that engage a neuron or sub-neuronal structure into a spatio-temporally extended fractal will most likely not be detectable by monitoring single neuron activity, unless the neurons are embedded into a supportive neuronal network. In future studies, we will investigate the possibility to simulate or create RFNNs in appropriate in vitro model systems, e.g. hybrid elements between neurons and electronic chips (Fromherz et al., 1991; Zeck and Fromherz, 2001). These neurochips are advantageous over classical electrode recordings due to noninvasive stimulation and monitoring of a neuronal network that has been naturally grown on a semiconductor surface. The neurochip setup allows for the correlation of network architecture with electrical activity, an analysis that cannot be achieved readily by single electrode recordings in brain slices. It will also provide the possibility to manipulate and analyze single neurons on the molecular level, e.g. by fluorescence labeling of membrane or cytoskeleton elements while monitoring or modulating the neuronal network activity. We expect that this approach will provide us with experimental evidence for the effects of a fractal network architecture or fractal, molecular lattice structure on neuronal network processing. 


\section{Acknowledgements}

The author is grateful to Drs Chris Nunn, Louis Irwin, and Ms Sarah MacKinnon, whose critical discussion of my work and invaluable suggestions inspired my motivation to keep on going with research on consciousness and improved the present manuscript significantly. The author also wishes to thank Dr. Robert K. Yu for his support and vision that research on the substrates of consciousness will be the future of neuroscience.

\section{References}

Adcock, R.A., Constable, R.T., Gore, J.C., Goldman-Rakic, P.S., 2000. Functional neuroanatomy of executive processes involved in dual-task performance. Proceedings of the National Academy of Science USA 97, 3567-3572.

Agmon-Snir, H., Segev, I., 1993. Signal delay and input synchronization in passive dendrite structures. Journal of Neurophysiology 70, 2066-2085.

Baars, B., 1997. In the theatre of consciousness. Journal of Consciousness Studies 4, 292-309.

Barnsely, M.F., 1993. Fractals Everywhere. Academic Press, Cambridge, MA

Beck, F., Eccles, J.C., 1992. Quantum aspects of brain activity and the role of consciousness. Proceedings of the National Academy of Science USA 89, 11357-11361.

Best, R.E., 1999. Phase-Locked Loops: Design, Simulation, and Applications. McGraw-Hill Companies, New York, NY.

Bieberich, E., 2000a. Principles of neural quantum computation and classical communication: darwinian evolution of the human mind in the quantum dynamical brain. 'Toward a science of consciousness', Tucson, 10th-15th April 2000. Consciousness research abstracts in Journal of Consciousness Studies, p. 88.

Bieberich, E., 2000b. Probing quantum coherence in a biological system by means of DNA amplification. Biosystems 57 , 109-124.

Borisyuk, R.M., Hoppensteadt, F.C., 1998. Memorizing and recalling spatial-temporal patterns in an oscillator model of the hippocampus. Biosystems 48, 3-10.

Cannon, R.C., Wheal, H.V., Turner, D.A., 1999. Dendrites of classes of hippocampal neurons differ in structural complexity and branching patterns. Journal of Comparative Neurology 413, 619-633.

Caserta, F., Eldred, W.D., Fernandez, F., Hausman, R.E., Stanford, L.R., Bulderev, S.V., Schwarzer, S., Stanley, H.E., 1995. Determination of fractal dimension of physiologically characterized neurons in two and three dimensions. Journal of Neuroscience Methods 56, 133-144.
Castet, E., Zanker, J., 1999. Long-range interactions in the spatial integration of motion signals. Spatial Vision 12, 287-307.

Chalmers, D., 1996. The Conscious Mind: In Search of a Fundamental Theory. Oxford University Press, New York, NY.

Davis, G.M., 1998. A wavelet based analysis of fractal image compression. IEEE Transaction on Image Processing 7, $141-154$

DeAngelis, G.C., Ghose, G.M., Ohzawa, I., Freeman, R.D., 1999. Functional micro-organization of primary visual cortex: receptive field analysis of nearby neurons. Journal of Neuroscience 19, 4046-4064.

Dragoi, V., Sur, M., 2000. Dynamic properties of recurrent inhibition in primary visual cortex: contrast and orientation dependence of contextual effects. Journal of Neurophysiology 83, 1019-1030.

Dufort, P.A., Lomsden, C.J., 1993. Cellular automaton model of the actin cytoskeleton. Cell Motility and the Cytoskeleton 25, 87-104.

Duggins, A., 2001. The mind/brain inequality. Biosystems 61, 95-108.

Freeman, W.J., Baird, B., 1987. Relation of olfactory EEG to behavior: spatial analysis. Behavioral Neuronscience 101, $393-408$.

Freeman, W.J., 1994. Role of chaotic dynamics in neural plasticity. Progress in Brain Research 102, 319-333.

Fromherz, P., Offenhausser, A., Vetter, T., Weis, J., 1991. A neuron-silicon junction: a Retzius cell of the leech on an insulate-gate field-effect transistor. Science 252, 1290-1293.

Gabor, D., 1968a. Holographic model of temporal recall. Nature 217, 584.

Gabor, D., 1968b. Improved holographic model of temporal recall. Nature 217, 1288-1289.

Gazale, M.J., 1999. Gnomon, From Pharaohs to Fractals. Princeton University Press, Princeton, NJ.

Greene, J.D., Sommerville, R.B., Nystrom, L.E., Darley, J.M., Cohen, J.D., 2001. An fMRI investigation of emotional engagement in moral judgement. Science 293, 2105-2108.

Hameroff, S., 2001. Consciousness, the brain, and spacetime geometry. Annual Reviews of the New York Academy of Science 929, 74-104.

Hameroff, S., Nip, A., Porter, M., Tuszynski, J., 2002. Conduction pathways in microtubules, biological quantum computation, and consciousness. Biosystems 64, 149-168.

Haykin, S., 1994. Neural networks. A comprehensive Foundation. Macmillan Coll. Publ. Comp, New York, NY.

Hoop, B., Peng, C.K., 2000. Fluctuations and fractal noise in biological membranes. Journal of Membrane Biology 177, $177-185$

Hopfield, J.J., 1982. Neural networks and physical systems with emergent collective computational abilities. Proceedings of the National Academy of Science USA 79, 2554-2558.

Hoppensteadt, F.C., 1989. Intermittent chaos, self-organization, and learning from synchronous synaptic activity in model neuron networks. Proceedings of the National Academy of Science USA 86, 2991-2995. 
Hoppensteadt, F.C., Izhikevich, E.M., 1998. Thalamo-cortical interactions modeled by weakly connected oscillators: could the brain use FM radio principles? Biosystems 48, 85-94.

Jefferys, J.G.R., Traub, R.D., Whittington, M.A., 1996. Neuronal networks for induced ' $40 \mathrm{~Hz}$ ' rhytms. Trends in Neuroscience 19, 202-208.

Jibu, M., Hagan, S., Hameroff, S.R., Pribram, K.H., Yasue, K., 1994. Quantum optical coherence in cytoskeletal microtubules: implications for brain function. Biosystems 32, 195-209.

John, E.R., 2001. A field theory of consciousness. Consciousness and Cognition 10, 184-213.

Koch, C., 1999. Biophysics of Computation: Information Processing in Single Neurons. Oxford University Press, New York, NY.

Koch, C., Segev, I., 2000. The role of single neurons in information processing. Nature Neuroscience 3, 1171.

Lindahl, B.I., Arhem, P., 1994. Mind as a force field: comments on a new interatomistic hypothesis. Journal of Theoretical Biology 171, 111-122.

Likenkaer-Hansen, K., Nikouline, V.V., Palva, J.M., Ilmoniemi, R.J., 2001. Long-range temporal correlations and scaling behavior in human brain oscillations. Journal of Neuroscience 21, 1370-1377.

Longuet-Higgins, H.C., 1968. Holographic model of temporal recall. Nature 217, 104-105.

Magee, J.C., Cook, E.P., 2000. Somatic EPSP amplitude is independent of synapse location in hippocampal pyramidal neurons. Nature Neuroscience 3, 895-903.

Mandelbrot, B.B., 1983. The Fractal Geometry of Nature. W.H. Freeman and Company, New York, NY.

Megias, M., Emri, Z.S., Freund, T.F., Gulyas, A.I., 2001. Total number and distribution of inhibitory and excitatory synapses on hippocampal CA1 pyramidal cells. Neuroscience 3, 527-540.

Menendez de la Prida, L., Sanchez-Andres, J.V., 2000. Heterogeneous populations of cells mediate spontaneous synchronous bursting in the developing hippocampus through a frequency-dependent mechanism. Neuroscience 97, 227241.

Miyashita, Y., Date, A., Okuno, H., 1994. Configurational encoding of complex visual forms by single neurons of monkey temporal cortex. Neuropsychologia 31, 1119-1131.

Nan, X., Jinghua, X., 1988. The fractal dimension of EEG as a physical measure of conscious human brain activities. Bulletin of Mathematical Biology 50, 559-565.

Orpwood, R.D., 1994. A possible neural mechanism underlying consciousness based on the pattern processing capabilities of pyramidal neurons in the cerebral cortex. Journal of Theoretical Biology 169, 403-418.

Peitgen, H.-O., Juergens, H., Saupe, D., 1992. Chaos and Fractals: New Frontiers of Science. Verlag, New York, NY.

Penrose, R., 1994. Shadows of the Mind: Search for the Missing Science of Consciousness. Oxford University Press, New York, NY.
Porter, R., Ghosh, S., Lange, G.D., Smith, T.G., Jr., 1991. A fractal analysis of pyramidal neurons in mammalian motor cortex. Neuroscience Letters 130, 112-116.

Pribram, K.-H., 1982. What the Fuss is all About. In: Wiber, K. (Ed.), The Holographic Paradigm and other Paradoxes. Shambala, London.

Psaltis, D., Brady, D., Gu, X.-G., Lin, S., 1990. Holography in artificial neural networks. Science 343, 325-330.

Rabouille, C., Cortassa, S., Aon, M.A., 1992. Fractal organization in biological macromolecular lattices. Journal of Biomolecular Structure and Dynamics 9, 1013-1024.

Radhakrishan, A., McConnell, H.M., 1999. Electric field effect on cholesterol-phospholipid complexes. Proceedings of the National Academy of Science USA 97, 1073-1078.

Reiter, C.A., 1994. Sierpinski fractals and GCDs. Computers and Graphics 18, 885-891.

Rucker, R., 1987. Mind Tools. Houghton Mifflin Company, Boston, MA.

Schroeder, M., 1991. Fractals, Chaos, Power Laws. W.H. Freeman and Company, New York, NY.

Searle, J.R., 2000. Consciousness. Annual Reviews in Neuroscience 23, 557-578.

Seife, C., 2000. Cold numbers unmake the quantum mind. Science 287, 791.

Singer, W., 2001. Consciousness and the binding problem. Annals of the New York Academy of Science 929, 123-146.

Sporns, O., Tononi, G., Edelman, G.M., 1991. Modeling perceptual grouping and figure-ground segregation by means of active reentrant connection. Proceedings of the National Academy of Science USA 88, 129-133.

Spruston, N., 2000. Distant synapses raise their voices. Nature Neuroscience 3, 849-895.

Stuart, G., Schiller, J., Sakmann, B., 1997. Action potential initiation and propagation in rat neocortical pyramidal neurons. Journal of Physiology 505 (Part 3), 617-632.

Sugar, I.P., Michonova-Alexova, E., Lee-Gau Chong, P., 2001. Geometrical properties of gel and fluid clusters in dmpc/ dspc bilayers: monte carlo simulation approach using a twostate model. Biophysical Journal 81, 2425-2441.

Tegmark, M., 2000. The importance of quantum decoherence in brain processes. Physical Reviews E 61, 4194-4206.

Teich, M.C., Heneghan, C., Lowen, S.B., Ozaki, T., Kaplan, E., 1997. Fractal character of the neural spike train in the visual system of the cat. Journal of the Optical Society of America A $14,529-546$.

Thomson, A.M., Bannister, A.P., 1998. Postsynaptic pyramidal target selection by descending layer III pyramidal axons: dual intracellular recordings and biocytin filling in slices of rat neocortex. Neuroscience 84, 669-683.

Triffet, T., Green, H.S., 1988. Information transfer by electromagnetic waves in cortex layers. Journal of Theoretical Biology 131, 199-221.

West, B.J., 2001. Fractal probability measures of learning. Methods 24, 395-402.

Woolf, N., 1999. Dendritic encoding: an alternative to temporal synaptic coding of conscious experience. Consciousness and Cognition 8, 447-454. 
Turcotte, D.L., Pelletier, J.D., Newman, W.L., 1998. Networks with side branching in biology. Journal of Theoretical Biology 193, 577-592.

Zeck, G., Fromherz, P., 2001. Noninvasive neuroelectronic interfacing with synaptically connected snail neurons im- mobilized on a semiconductor chip. Proceedings of the National Academy of Science USA 98, 10457-10462.

Zeki, S., 2001. Localization and globalization in conscious vision. Annual Reviews of Neuroscience 24, 57-86. 